\title{
Lapurdum
}

LAPURDUM Euskal ikerketen aldizkaria | Revue d'études basques |

Revista de estudios vascos | Basque studies review

$19 \mid 2016$

Numéro XIX

\section{Trovador vasco}

\section{Gorka Aulestia}

\section{OpenEdition \\ Journals}

\section{Edición electrónica}

URL: https://journals.openedition.org/lapurdum/3146

DOI: 10.4000/lapurdum.3146

ISSN: 1965-0655

Editor

IKER

Edición impresa

Fecha de publicación: 1 enero 2016

Paginación: 67-83

ISBN: 978-2-95534-134-6

ISSN: $1273-3830$

Referencia electrónica

Gorka Aulestia, «Trovador vasco», Lapurdum [En línea], 19 | 2016, Publicado el 01 enero 2021, consultado el 03 septiembre 2021. URL: http://journals.openedition.org/lapurdum/3146 ; DOI: https:// doi.org/10.4000/lapurdum.3146

Creative Commons - Attribution - Pas d'Utilisation Commerciale - Pas de Modification 4.0 International - CC BY-NC-ND 4.0 


\title{
Trovadores vascos
}

\author{
Gorka AULESTIA
}

\section{Proyecto cultural y político de Aitzol (1927-1936). Euskal Pizkunde (Renacimiento cultural vasco)}

\subsection{Aitzol (1896-1936)}

José de Aiztimuño, Aitzol, desplegó una actividad cultural poco común en el Renacimiento vasco previo a la Guerra Civil Española (1927-1936), como árbitro de la belleza literaria y promotor de muchos jóvenes escritores vascos de esa época. Entre ellos se hallaba J. Zaitegi quien calificó a la generación de este hombre singular como "la generación de Aitzol". Fue sin duda alguna, el personaje clave en el País Vasco en la década 1926-1936, y muy especialmente, durante los cinco años que duró la II República Española (1931-1936) por el papel jugado en el Renacimiento cultural vasco. Nació el 18 de marzo de 1896 en Tolosa (Gipuzkoa); fue fusilado por las tropas franquistas el 18 de octubre de 1936 y se le enterró en una fosa común con otros sacerdotes vacos junto a los muros del cementerio de Hernani, pero fuera del lugar sagrado.

Desde joven se mostró interesado y preocupado por el devenir de "las naciones sin estado" y por la restauración del euskara en el País Vasco mediante la cooficialización de esta lengua y la implantación del bilingüismo. Para ello, viajó en 1927 a Lovaina (Bélgica) para conocer "in situ" los problemas que creaba el bilingüismo. Pero el proyecto de Aitzol no era simplemente cultural sino también político. En consecuencia, se interesó del nacimiento de las nuevas naciones tras la desaparición del Imperio Austro-Húngaro después de la I Guerra Mundial (1914 -1918). En lo relativo al País Vasco, se mostró siempre convencido de que la lengua y literatura vascas (especialmente la poesía) estaban íntimamente ligadas a la lucha por la consecución de la autodeterminación, soberanía e independencia de Euskal Herria. Aquellas eran la mejor palanca para que una nación lograra su propia identidad.

Aitzol (al igual que Lizardi y la mayoría de los escritores de aquella generación) pensaba que el poeta era un hombre providencial en el resurgimiento de los pueblos, y el artífice de la supervivencia de las lenguas poco cultivadas; idea romántica muy extendida en el s. XIX por toda Europa, ávida del "couleur locale romantique" y del fomento de las lenguas minoritarias 
(propugnadas por los escritores del Romanticismo). Así por ejemplo, Frédéric Mistral (18301914) Premio Nobel de literatura en 1904, había logrado que una lengua minoritaria como el provenzal alcanzara un prestigio internacional gracias a su obra Mirèio. Por ello, en 1930 (primer centenario del nacimiento de F. Mistral) Orixe publicó la versión vasca de Mirèio (1930), a instancias de Aitzoll. Este como otros muchos vascos, estaban al corriente además de un Congreso Internacional sobre Artes Populares celebrado en 1928 en Praga, como se desprende de los bertsos de Basarri.

$\begin{array}{ll}\text { Bertsolariak eman diote } & \text { Los bertsolaris han ensalzado } \\ \text { Txeko erriari gorantza } & \text { al pueblo checo } \\ \text { "Mireio" batek edergallutan } & \text { un Mireio } \\ \text { yosia zuan Provenza.2 } & \text { llenó de esplendor a Provenza. }\end{array}$

Pero, para lograr los fines culturales y socio-políticos de éste, no era suficiente una mera traducción de un libro, por muy laureado que hubiera sido su autor. Era necesario que un poeta vasco (Orixe, según Aitzol) escribiera una gran epopeya vasca que ensalzara la nación e hiciera brillar al euskara: Euskaldunak (Los Vascos) finalizada por Orixe en 1936 pero publicada en 1950, por razones ajenas a su voluntad (guerra, cárcel, etc.).

Por otra parte, en 1930, el poeta vizcaíno Lauaxeta había sido galardonado por su poesía "Maitale Kutuna" (La amada predilecta) en el I Olerti Eguna ("I Día de la Poesía Vasca") celebrado en Errentería (Gipuzkoa); y en 1931, Lizardi conseguía igual premio en Tolosa en el II Olerti Eguna con su "Baso Itzal" (Bosque sombrío), dedicada a una de las cuatro estaciones del año de su poema "Urte giroak ene begian", (Las estaciones del año en mi pupila) (1930). En los primeros años de "Pizkundea" Aitzol abogó a favor de una poesía culta que alcanzara la calidad y el nivel de la poesía de otros países europeos. Pero este influyente árbitro (en ocasiones hasta impositivo) que había premiado (junto con otros miembros del jurado) estas dos obras de bella textura pero que no calaban en el pueblo (entre otras razones, porque sus publicaciones no pasaban de 300 ejemplares) comenzó a impacientarse por la desorientación (según él) de algunos poetas vascos. En 1932, condenó las dos poesías de sus dos amigos: Lizardi y Lauaxeta por ser difíciles para la compresión de la mayoría de los vascos, por el hermetismo y vanguardismo que mostraban estos textos. Además, la fecha de 1932 supone algo más que esta condena pues representa también el comienzo de un período de crispación en torno a la clase de poesía vasca que se debía crear. ¿Se tenía que escribir una poesía elitista, moderna, conceptual y difícil para la mayoría, o un tipo de poesía narrativa y tradicional, cercana a la poesía oral y popular de los bertsolaris?

En 1933, con ocasión del "IV Día de la poesía vasca" celebrado en Urretxu (Guipúzcoa) en honor del bardo J.M. Iparragirre, el tribunal premió la sencilla poesía "Bost lore" (Cinco flores) del P. Etxeberria S. J., de estructura afín a una "Kopla zaharra" (Vieja copla) estilísticamente muy diferente de las dos premiadas en 1930 y 1931. En un artículo publicado en El Día en febrero de 1933, Aitzol condenó sin paliativos la poesía difícil de sus dos amigos confesando su culpabilidad.

Hemos aburrido a los lectores y a los amantes de lo vasco con nuestros versos difíciles e incomprensibles [...] yo soy el culpable [...] llevamos mal camino los poetas y la mayoría de los escritores. 2 
El año 1934 marca un nuevo jalón en el cambio operado en Aitzol y escribe en su revista Yakintza un artículo ("Euskal Olertikera berezia (estetika") sobre la teoría de la poesía popular vasca en el que defiende la existencia de una estética genuina en la literatura tradicional vasca.3 Su pesimismo llega al cénit transformándose en decepción como se puede comprobar en una carta que escribió el fin de año de 1934 a su amigo, J. Zaitegi, exiliado en Marneffe (Bélgica) donde residían los jesuitas desterrados por el gobierno de la II República (1931-1936).

\footnotetext{
Con el euskara que estamos produciendo, estamos hastiando a los lectores. Los libros no se venden. Estamos haciendo una literatura artificial. No sabemos bajar a las entrañas del pueblo, y los del pueblo, huyen; es de verdad lamentable lo que nos está ocurriendo. Cada vez se habla menos en euskara; y se lee menos también. 4
}

Finalmente en el año 1935, al hacer el balance de los libros publicados en 1934 hace un elogio de tres obras Itz-lauz (En prosa, 1934) y Umezurtz Olerkiak (Poesías huérfanas, 1934) de Lizardi y Barne Muinetan (En las médulas del interior, 1934) de Orixe, pero resaltando al final los aspectos negativos (según él) de estas obras.

\footnotetext{
Estos tres tomos, a pesar de su indiscutible valor literario, no llegarán, casi podemos asegurarlo, a robustecer lo esencial, el euskara. Fáltales lo sencial, la frescura, la naturalidad de un euskara fluido, fácil, inteligible [...].5
}

Ya no le quedaba más que un paso para distanciarse de los poetas innovadores y animar la poesía oral de los bertsolaris, apoyándose en la obra de M. Lekuona, Literatura oral euskérica (1935). Para ello contribuyó activamente en la organización del "I Día del Bertsolari" (I Txapelketa Nacional) celebrado el 20 de enero de 1935 en el teatro "Poxpolin" de Donostia bajo los auspicios de las asociaciones "Euskaltzaleak" (Amigos del Euskara) y "Eusko Gaztedi" (Juventud Vasca). Tomaron parte veinte bertsolaris y salió vencedor el joven Basarri.

\subsection{Manuel Lekuona (1894-1987)}

Este insigne oralista y poeta que dedicó más de setenta años de su vida al estudio, pudo ponerse desde muy joven al corriente de la cultura vasca gracias a la biblioteca de su tío sacerdote Miguel Antonio Iñarra (1864-1898) en su pueblo natal de Oiartzun (Guipúzcoa).6 Cultivó los géneros de poesía, teatro y ensayo publicando trabajos de exigencia académica anteriores a la Guerra Civil Española entre los años 1918 y 1936 y los trabajos de investigación y divulgación realizados durante la posguerra en diversos campos de la cultura vasca (etnografía, arte, historia, etc), llegando a publicar doce volúmenes: Idazlan Guztiak. Estudió en el Seminario de Vitoria siendo ordenado sacerdote en 1916. Un año antes de su ordenación obtuvo la cátedra de euskara en el mismo centro en el que enseñó hasta el estallido de la Guerra Civil en 1936. El joven profesor era desde niño entusiasta del bertsolarismo y dio una conferencia sobre "Métrica Vasca" en el Seminario de Vitoria con ocasión de la apertura del curso académico de 1918 .

Pero lo que realmente le animó a dedicarse al campo de la literatura oral y al bertsolarismo fueron las palabras despreciativas de un periodista de S. Sebastián en una conferencia pronunciada en Oñate con ocasión del I Congreso de Eusko Ikaskuntza celebrado del 1 al 18 de 
setiembre de 1918. El mencionado reportero, describió a los labriegos alemanes que escuchaban en las cervecerías los bellos poemas de J. W. Goethe (1749-1832) y J. C. F. Schiller (1759-1805) mientras que los "baserritarrak" en el País Vasco se reunían en las sidrerías para oír las simplezas de los bertsolaris. Como él mismo lo confiesa: "La calificación de simplezas, aplicada a nuestros bardos populares me dolió en el alma. Y puedo decir que desde entonces concebí el propósito y plan de dedicarme a montar una Apología del Bertsolarismo".7

Tras varios años de reflexión sobre las distintas expresiones de la literatura oral, M. Lekuona dio una de sus conferencias más importantes en el mes de setiembre de 1930 en Bergara, con ocasión del V Congreso de Eusko Ikaskuntza. El título de dicha conferencia fue, "La poesía oral vasca", y la dividió en tres partes: la poesía decorativa, las "Kopla Zaharrak" (viejas coplas) y el bertsolarismo. En esta última parte, el autor analiza este fenómeno presentando una visión teórica de la oralidad vasca con consecuencias interesantes para la creación literaria. El impacto producido por esta nueva teorización poética en Aitzol fue muy fuerte y desde entonces apostó a favor de un bertsolarismo distinto al de las sidrerías (únicas "universidades" de los vates vascos como "Txirrita"), que sirviera para su proyecto cultural. Sus palabras pronunciadas son significativas.

¡Qué espectáculo más bochornoso cuando el día de Santo Tomás [...] dos bertsolaris de voz desagradable, de una increíble plebeyez de concepto, de frase atrevida y de mal gusto, de versos más elásticos que la goma y mal medidos, actuaron en el teatro Bellas Artes!.8

El año 1935 es señalado como clave en la historia del bertsolarismo por dos razones: la celebración del "I Día del Bertsolari” y por la publicación del libro Literatura oral euskérica, fruto maduro de las investigaciones de juventud de M. Lekuona y, sobre todo, de su mencionada conferencia de Bergara.

Además de estas razones, resulta también interesante esta obra por la influencia ejercida en ella por el libro Le Style oral rhythmique et mnémotechnique chez les verbo-moteurs (1925) del oralista y profesor de la Sorbona, Marcel Jousse, (1886-1962). Fue publicado diez años antes en 1925, y se abordan en él, entre otros temas, los problemas del lenguaje analizando la espontaneidad del gesto humano. Partiendo de las vivencias experimentadas en su niñez, enriquecidas con los relatos orales contados por su madre y abuela (iletradas pero no incultas), M. Jousse se cuestiona la hegemonía de nuestra cultura (basada esencialmente en la escritura), que esta sociedad intenta imponer al hombre como única vía de civilización. Posponiendo la filosofía idealista dominante entonces en las universidades francesas, M. Jousse profundizó en la formación campesina y relativizó la cultura greco-latina, porque aquella ocultaba una realidad más rica y profunda. Este jesuita francés, antiguo militar en la I Guerra Mundial (1914-1918), afirmaba que el ser humano se manifiesta no solo a través de la boca sino por medio del cuerpo, especialmente de sus manos. Además, contrastaba sus experiencias infantiles con algunos pasajes de la Biblia y de las dos epopeyas de Homero: la Iliada y la Odisea, concluyendo que en estos libros existían unas fórmulas y frases estereotipadas o clichés tomados de la tradición oral y popular.

Aunque M. Lekuona llevaba ya varios años sumergido en el estudio de la literatura oral y popular, el libro del profesor francés marcó una referencia muy importante en su pensamiento. Además de mencionar a M. Jousse varias veces, M. Lekuona comenta varios puntos expuestos 
por el profesor de la Sorbona: la importancia de la memoria en la literatura oral; las diversas fuentes que usaron Homero y Virgilio, y las diferencias de estilo entre estos dos autores clásicos; la importancia de las reuniones campesinas de su pueblo en las que se cantaban y recitaban pasajes sin leerlos previamente; la mención de algunos personajes bíblicos reseñables por su capacidad para la improvisación poética, etc.

Como conclusión de esta parte dedicada a M. Lekuona, nada mejor que leer las líneas con las que su sobrino, Juan $\mathrm{M}^{\mathrm{a}}$ Lekuona, poeta y oralista le describe:

\begin{abstract}
A la hora de señalar su especialidad más característica diríamos que se le consideraba como la figura señera de los oralistas vascos. [...] Poseía su experiencia en trabajos de campo. Conocía como pocos la tradición oral viva y la memoria colectiva. Y además, dedicó muchos años al estudio de la documentación local, hurgando en archivos [...]. Manuel Lekuona nos enseñó a ser cultos desde las formas locales pero sin perder de vista la perspectiva universal. Nuestra cultura comienza por lo que nos rodea más inmediatamente. Aquí se concreta la herencia que nos legó Don Manuel y el mensaje callado de su vida y de su obra.9
\end{abstract}

\title{
1.3. Iñazio Eizmendi, Basarri (1913-1999)
}

Otra de las personas con las que Aitzol contó para llevar a cabo su proyecto cultural fue el joven bertsolari Basarri. En 1935, M. Lekuona había secundado este proyecto con su libro y la participación como miembro del jurado en el "I Bertsolari Eguna" celebrado en Donostia; en ese mismo concurso, un joven de 22 años, nacido el 27 de noviembre de 1913 en Errezil (Gipuzkoa) pero afincado desde los siete años en Zarautz (Gipuzkoa) se convirtió en el bertsolari paradigmático deseado por Aitzol y muchos nacionalistas al proclamarse campeón del certamen. El anciano y patriarca del bersolarismo tradicional, Txirrita (a sus 75 años), con más de $120 \mathrm{~kg}$. de peso y vestido con una blusa tradicional negra no servía para llevar a cabo a buen puerto los planes de Aitzol. Hacía falta un bertsolari que renovara los temas y recursos de este fenómeno artístico. En opinión de Juan M. Lekuona, (uno de los mejores especialistas de este fenómeno literario), gran conocedor de la obra de Basarri, este se convirtió no solo en campeón sino también en pionero y portador de un nuevo proyecto que marcaba una nueva escuela, al sacar el bertsolarismo de las sidrerías y llevarlo a las plazas.

\footnotetext{
"Lehenengo Bertsolari Eguna" 1935ean ospatu zenean, Basarrik saria irabazi ezezik, aintzindaritza bat hartu zuen bere bizkar, bertsolaritza berriagoan aurresku izateko eginkizuna hartu. Eta gero, urte askotan gainera, zeregin hori bete bertsozko emanaldietan, batez ere plazako eta jendaurreko bertsolaritzan.10
}

Dentro del proyecto cultural de Aitzol, Basarri contribuía también con su proyecto personal sobre este fenómeno artístico que fue describiéndolo en revistas y diarios en años sucesivos. Esta nueva "escuela" no renegaba del pasado del bertsolarismo; antes al contrario, tenía en gran estima la obra de Bilintx, Pedro Ma Otaño y K. Enbeita. Pero Basarri consideraba que había llegado la hora final del bertsolarismo de sidrerías (Udarregi, P. Errota, etc.) cuyo representante principal era, a la sazón, el anciano Txirrita. Siendo aún niño, Basarri había conocido de cerca esta expresión literaria gracias a los mejores bardos de entonces que 
frecuentaban el bar "Azken-Portu" de sus padres en Zarautz. Según sus propias palabras, para los doce años de edad, estaba convencido de la urgente necesidad de una renovación profunda de este fenómeno literario. Los bertsolaris, a pesar de sus buenas cualidades para la improvisación de bertsos, estaban errados al haber elegido el peor de los caminos. En vez de atraer a los oyentes los alejaban por el torpe lenguaje que usaban.

\begin{abstract}
Amabi urte nituanerako konturatu nintzan ortaz. Etxean taberna genduan eta garai artan pamarik geien zuten bertsolariak an kantatuak ziran [...] biderik kaxkarrena aukeratu zuten beren kaltetan [...]. Entzuleak erakarri bearrean uxatu egiten zituzten beren izketabide trakets eta merkearengatik.11
\end{abstract}

En su proyecto bertsolarístico, la ilustración y cultura jugaban un papel importante a través de la lectura de los libros. Había que superar la etapa en que la mayoría de los bertsolaris no supieron ni leer ni escribir, o (en el mejor de los casos) solo sabían leer como Xenpelar, Txirrita y la inmensa mayoría de los bardos vascos del s. XIX y comienzos del XX.12. Por ello, el autodidacta Basarri (mediante el esfuerzo y la lectura) consiguió una formación suficiente para convertirse en periodista y locutor de radio en San Sebastián. Cultivó el periodismo (su afición favorita) durante cuarenta años escribiendo a menudo en revistas y periódicos sobre deportes rurales y bertsolarismo: Zeruko Argia, Aránzazu, Anaitasuna, Gudari, Euzkadi, Goiz-Argi, Argia; Eguna, El Día, La Voz de España, El Diario Vasco, La Hoja del Lunes, etc. Además, escribió varios libros; seis en verso y el séptimo en prosa: Atano III: bere edestia bertsotan (1949); Basarriren Bertso-Sorta (Ramillete de estrofas de Basarri, 1950); Kantari nator (Mi cancionero, 1960); Laugarren Txinpartak (Cuarto libro de poesías, 1966); Sortu zaizkidanak (Mis creaciones, 1973); Kezka giroan (En medio de inquietudes, 1983); Bertsolaritzari buruz (En torno al Bertsolarismo, 1948). Nere bordatxotik (Desde mi cabaña, 1992).13

Ante semejante producción, el lector podría preguntarse sobre la escuela, instituto o universidad que frecuentó Basarri en la juventud. Aunque la siguiente cita puede resultar larga, nada mejor que escuchar sus propias palabras para salir de dudas y evitar falsas interpretaciones sobre los orígenes culturales de este bardo ilustrado y roturador de nuevos caminos de esta poesía oral improvisada. La cita nos servirá también para constatar el grado tan lamentable en que se hallaba el bertsolarismo.

[...] "Basarri", desgraciadamente, es un ignorante en solfeo y música; incapaz de leer las notas del pentagrama. También andan equivocadísimos quienes me creen un hombre culto, un hombre de formación, un intelectual. [...] Sepan todos los que me leen que "Basarri" solamente pisó la escuela hasta los once años de edad; que tuvo que abandonarla forzosamente al hacer su primera comunión para atender a las necesidades de la familia [...]. Sufría horrores con el bajo e intolerable lenguaje que empleaban los antiguos bertsolaris en las plazas públicas. No acertaban a versificar sino atacándose mutuamente y en tonos muy groseros e impertinentes. Las borracheras, los latrocinios, los adulterios eran los temas de aceptación. Cuanto mayores disparates soltaban, más celebraban los oyentes. Pero la gente de respeto, la gente de sentido común, la gente un poco culta, huía del bertsolari. Este pertenecía a la última escala de la sociedad. He podido comprobar que aún existen quienes opinan que el verso tiene que ser eso; que el bertsolari ha de ser una especie de bufón, el hazmerreír de las gentes.14 
Mientras muchos pensaban que el bertsolari era una especie de payaso de circo cuya misión era hacer reír a la gente fomentando así el bertsolarismo de humor (en el que Txirrita era un gran maestro), el joven Basarri iba forjando poco a poco su proyecto robando muchas horas al sueño para adquirir una cultura.15 A diferencia de la cigarra ("Txirrita" en euskara) que pasaba noches enteras en las sidrerías, la "hormiga" de Zarautz trabajaba sin descanso logrando poco a poco sus objetivos.

Lengo denbora aietan sagardotegiak ituan bertsolari eskola edo akademi.16

(En el pasado las sidrerías se convirtieron en escuela y academias de bertsolarismo). Trataba de lograr un euskara popular pero elegante siguiendo por el surco trazado por P. M. Otaño, sin los "purismos" dominantes. Se buscaba una renovación en las melodías y las estrofas; un euskara sin tantas palabras castellanas como usaban Udarregi, Txirrita, etc.; temas más actuales y más interesantes para atraer a un público mayor mediante los medios de comunicación (prensa y radio); actuaciones mas cortas para evitar el tedio entre la gente. En cuanto a las estrofas, debían ir bien entrelazadas evitando el "bete lana" o cantar versos de relleno sin decir gran cosa. El bertsolari no se debía limitar a elaborar con esmero el verso final buscando así el aplauso del público, sino que el final del verso debía ser el colofón de una estrofa bien urdida y concatenada siguiendo el hilo de una narración lógica.

Ori guzia betelena da [...]. Bertso onak, bertso mardul, oso ta bikañak, eztu olakorik izaten. Asieratik bukaeraño izaten da giartsua. Naiz azkenengo puntuan bere gatz eta piperrra izan, era ontan moldatutako bertsoa ezta txalogarri.17

En cuanto al punto de las rimas y el tipo de estrofas como el "bederatzi puntukoa" (estrofa de 14 versos y 9 rimas), Basarri no era partidario de agravar más la situación imponiendo en los concursos esta difícil estrofa a los bertsolaris. Ello contribuiría a ponerlos en aprieto y a estropear el espectáculo.

\footnotetext{
Emengo bertsolariak geien ondatzen gaituna puntu legea da [...] Gaiak ezarri ta bederatzi puntuko bertsoak aurrez aurre kantatzera beartu bertsolariak. Bertsolariak keldotzeko, larriak ugaltzeko, ta jaialdiak moteltzeko eztiteke xixtima oberik autatu. 18
}

Una de las ideas básicas de este proyecto es, sin duda alguna, su definición del bertsolari. El vate vasco es, según Basarri, simplemente un poeta popular. Este adjetivo calificativo marca la diferencia y le distingue del poeta que escribe poesía sin improvisarla y sin cantarla ante un público. La controversia mantenida con Gabriel Aresti prueba a las claras los diferentes puntos de vista que existían entre ambos. Según el poeta bilbaíno, el bertsolari es el mejor poeta entre todos los poetas tanto populares como los que escriben poesía.

Artikulu labur honetan gauza bakar bat esan diezaioket Basarriri, bertsolariak ere poetak direla, poeta baserritarrak [...] eskolarik gabeko poetak, baina nire iritzian euskaldun poetarik hoberenak, olerkari guztiak baino hobeak.19 
Basarri no compartía en absoluto estas ideas, y aun aceptando que el bertsolari pudiera estar más cerca del pueblo que el poeta y ser más útil al euskara, la afirmación de G. Aresti, según Basarri, equivaldría a decir que los mejores pescadores son los mineros:

"Arrantzalerik onenak mineroak dirala esatea añakoa da ori".20

Basarri dedicó su larga vida al fomento y difusión de la cultura popular vasca, especialmente del bertsolarismo. Supo llevar con dignidad y valentía el testigo que se le encomendó en 1935. Tras sufrir como perdedor las consecuencias de la Guerra Civil (exilio, cárcel, trabajos forzados, etc.) pudo por fin en 1942 proseguir actuando como bertsolari en compañía de su compañero y amigo, el tres veces campeón Uztapide. Basarri ganó también el primer puesto en la "Txapelketa Nacional" de 1960, y el segundo premio en la de 1962. Desde entonces, decidió no participar más en estos concursos aunque en 1968 obtuvo el primer puesto, compartido con Xalbador, en un certamen importante organizado por "Piensos Onena" en Donostia. Murió con 86 años casi cumplidos el 4 de noviembre de 1999. Con su muerte el País Vasco perdió a uno de los bertsolaris más completos de su historia y desapareció un mito del bertsolarismo.

\section{La década de los 60. Uztapide y Xalbador}

\subsection{Manuel Olaizola, Uztapide (1909-1983)}

No sería justo detenernos en la explicación del proyecto de Basarri sin citar el nombre de un bertsolari de los más queridos como persona (por su sencillez) y más apreciados (como artista) por lo que diremos a continuación. El tándem Basarri-Uztapide fue la primera pareja (más tarde vendrían Xalbador-Mattin y J. Lopategi-J. Azpillaga) que en la década más dura del franquismo (1940-1950), fue avivando entre las brasas el rescoldo de la identidad vasca que aún perduraba tras tres años de cruel guerra (1936-1939). Esta pareja de vates guipuzcoanos recorrió en un cuarto de siglo prácticamente el País Vasco, comenzando desde su provincia natal y llegando hasta Iparralde (zona norte del País Vasco) donde tomaron parte en muchas competiciones junto a Xalbador, Mattin, etc. entre los años 1949-1958. Nos lo confirma el propio Basarri:

Ogeitaka urtetan ibili giñan lagun "Uztapide" ta ni Euskalerria serbitzen. Gelditu gabe ara ta onera.21

En una época en la que el uso del euskara estaba prohibido en actos públicos excepto en las iglesias, la presencia de estos dos bertsolaris era también imprescindible en el programa festivo de algunos pueblos. Ambos eran muy diferentes como personas y como artistas. Posiblemente por esas grandes diferencias a la hora de expresar su arte, fueron capaces de formar una pareja ideal, requerida y apreciada en tantos pueblos. Personalmente, Basarri era un hombre de talante más bien serio, mientras que Uztapide era una mezcla del pícaro y trasnochador Txirrita (capaz de pasar una noche entera improvisando versos y bebiendo) y del fiel "baserritarra" o aldeano entregado a las faenas de su caserío. Una fina ironía unida a un humor no hiriente y a una aguda inteligencia fueron tres de sus armas dialécticas más importantes. A lo largo de los años, el primero se mostraba más conservador, mientras 
que el segundo aparecía siempre más cercano a los jóvenes y más popular. En el Terrero bertsolarístico Basarri superaba a Uztapide en la variedad de ritmos y melodías (algunas creadas por él mismo), mientras que el segundo usaba preferentemente los "zortziko" (estrofa de ocho versos) y "hamarreko" (estrofa de diez versos). Ambos fueron excepcionales; Uztapide obtuvo tres campeonatos nacionales (en 1962, 1965 y 1967) y Basarri, dos (en 1935 y 1960). Cuando actuaban en pareja se complementaban bien; este era como una especie de arado que roturaba y preparaba el terreno para que su compañero Uztapide comenzara a sembrar. Es de destacar su capacidad en las competiciones para no cortar el hilo dialéctico de su partenaire. Basarri se mostraba muy cuidadoso con las formas gramaticales y retóricas llegando a ser uno de los bertsolaris que menos bertsos incorrectos ha improvisado en la historia moderna de este fenómeno. El arte bertsolarístico de Uztapide, en cambio, no era tan retórico al no ser su autor tan ilustrado como aquel. Pero puesto a improvisar versos era como un caudal que no se agotaba. Se asemejaba al personaje de Martín Fierro a quien las coplas le salían como agua de manantial:

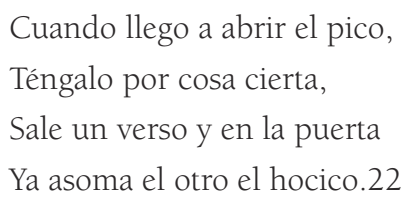

Mientras Basarri se retiró a los 49 años (1913-1962) de las competiciones nacionales, Uztapide continuó con otros compañeros como M. Lasarte, Lazkao-Txiki, etc. hasta que se vio obligado a retirarse por enfermedad.

\subsection{Fernando Aire, Xalbador (1920-1976)}

El estallido de la Guerra Civil Española (18 de julio de 1936) interrumpió la vida cultural vasca sometiéndola a un gélido invierno de casi cuarenta años. El bertsolarismo no se libró de las nocivas consecuencias provocadas por esta terrible tormenta aunque hubo algunas épocas de cierta calma. Tras casi un cuarto de siglo (1936-1960) se reanudaron los campeonatos nacionales en la década de los 60 en los que Xalbador tomó parte como finalista tras varias eliminatorias: cuarto puesto en 1960; tercero en 1962 y en 1965; y segundo en 1967. En algunas de estas competiciones improvisó varias estrofas antológicas que mencionaremos más tarde. Asimismo, logró el segundo puesto en un campeonato muy importante organizado en 1968 por "Piensos Onena" en Donostia. En el terreno de los "bertso-paperak" o versos escritos obtuvo cuatro primeros premios en el concurso "Xenpelar Saria" de Errenteria en los años 1972, 1973, 1975 y 1976; fue asimismo premiado en los concursos "Mendaro Txirristaka"y "Donostiako Kutxa Saria".

Xalbador fue, sin duda, el mejor bertsolari transpirenaico; se declaraba "citoyen Français" por su pasaporte y euskaldún de corazón. Se le considera como uno de los mejores bardos de toda la historia del bertsolarismo. El lugar de su nacimiento nos obliga a situar el bertsolarismo como un fenómeno literario ubicado cerca de los Pirineos Occidentales, tal y como hizo Voltaire en el siglo XVIII al describir a los vascos como pueblo que bailaba junto a estas montañas: "Ces peuples qui sautent au pied des Pyrénées et qu'on appelle Vasques ou Vascones".23 En el bello pueblecito bajo-navarro de Urepel, situado entre altas montañas 
nació Fernando Aire el 19 de junio de 1920.

Desde niño dio muestras de su fuerte personalidad pues a los once años abandonó la escuela francesa en la que no le enseñaban en euskara. A los dieciséis se hizo cargo del rebaño de ovejas en la alta montaña junto a la cabaña, su amada, a la que dedicará más tarde algunas de sus estrofas líricas más hermosas; a esa edad se atrevió a improvisar una estrofa por primera vez ante algunas pocas personas. A los diecinueve, cuando ya se sentía preparado para saltar a la plaza pública, el posible inicio bertsolarístico se truncó por el estallido de la II Guerra Mundial (1939-1945) y por la muerte de su padre. En 1946 conoció en San Juan de Luz a Mattin, su "escudero", con quien formó pareja para las actuaciones bertsolarísticas durante treinta años (1946-1976).

Xalbador es un bertsolari atípico por su dialecto bajonavarro al que la inmensa mayoría de los oyentes no estaba familiarizado; la novedad de sus melodías desconocidas hasta entonces en el País Vasco peninsular; por la finura y sensibilidad poética que imprimía a sus versos; por la hondura de sus pensamientos; por el lirismo que emana de varias de sus mejores estrofas; por su aportación en el terreno del estrofismo con nuevos ritmos, siguiendo el ejemplo de Xenpelar en el siglo XIX (pensemos por ejemplo en el largo verso de 18 sílabas $(5+5+8)$ de su "Esperantzarik gabeko amodioa"); por la capacidad para expresar bellamente los temas más triviales; por algunos de sus versos improvisados que entrarían con seguridad en la mejor antología, y finalmente, por la belleza de sus bertsos escritos, algunos de los cuales rayan a un nivel poético muy alto. Los hallamos, al menos, en tres de las cuatro "txapelketak", publicadas en la colección Auspoa (nº 22 (1962), no 43 (1965) y nº 67 (1967), así como en sus tres libros: Ezin bertzean (A la fuerza, 1969), Herria gogoan (Con la mirada puesta en el Pueblo, 1981) y, especialmente, en Odolaren mintzoa (El lenguaje de la sangre, 1976); en este último resaltaría varios de sus bertsos escritos como "Esperantzarik gabeko amodia" (El amor sin esperanza), "Jainkoa eta ni" (Dios y yo), "Gure artzain etxolari" (A nuestra cabaña pastoril), "Ortzirale saindu batetako gogoetak eta otoitza" (Reflexiones y oración de un Viernes Santo), etc.

Entre los bertsos improvisados escogería tres como simples ejemplos del arte bertsolaristico de Xalbador: "Emazte il zanaren soñekoari" (Al vestido de tu difunta mujer), tomado de la "txapelketa" nacional de 1965 (pp. 93-94); "Bazkaria serbitu dizun neskatxari" (A la camarera que te ha servido la comida), improvisando en la "txapelketa" de 1967, p.71 y "Ohea" (La cama), igualmente repentizado en la misma competición (pp. 119-120). Esos bertsos dedicados al vestido de su difunta mujer muestran la habilidad de Xalbador a la hora de dialogar con objetos como si fueran personas animadas.

\author{
Pentsa zazute alargun bat \\ ez daiket izan urusa, \\ dolamen hunek, oi! ez dezala \\ ainitz gehiago luza, \\ orai urte ziloan sartu \\ andereñoaren gorputza, \\ haren arropa hantzet dilindan \\ penaz ikusten dut hutsa.
}

Geroztik nihaur ere nabila guzia beltzez jantzirik

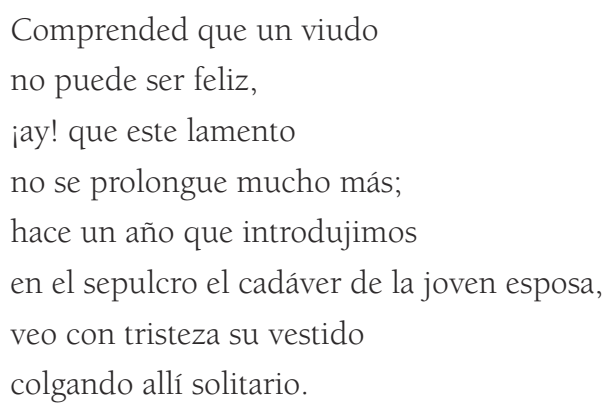

Desde entonces yo vivo

vestido de luto riguroso, 


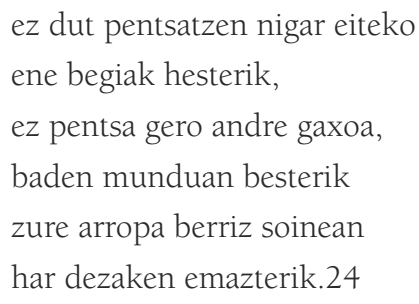

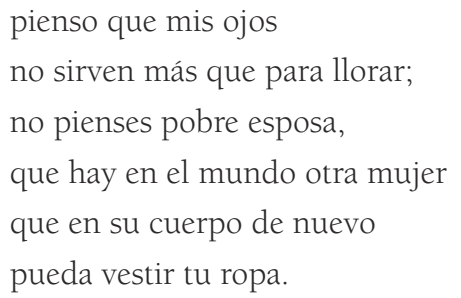

Otra de las cualidades con las que Xalbador sorprendía a los oyentes era su capacidad para saber adaptarse al momento y lugar requeridos, algunas veces con símiles y metáforas. En un certamen celebrado el 6 de enero de 1962 (Festividad de los Reyes Magos) en San Sebastián, Xalbador (como los tres magos de Oriente) se apresura (azkarra) a hacer un viaje (tuvo que salir muy temprano de su pueblo, Urepel), y se topa con una estrella (izarra) en la ciudad de Donostia que nos evoca a la Jerusalén sobre la cual se detuvo la estrella ("Donostiren par-parean gelditu zeraut izarra").

\author{
Egun Erregen eguna da ta \\ nik ere hartu indarra \\ nunbait ni ere Erregen gisa \\ bidean asi bearra, \\ goizean etxetik erten eta \\ abiatu naiz azkarra, \\ gero Donostiren par-parean \\ gelditu zeraut izarra. 25
}

\author{
Puesto que hoy es festividad de Reyes \\ también yo he tomado la decisión \\ de ponerme en camino \\ como lo hicieron ellos, \\ he salido de casa por la mañana \\ y he partido rápidamente, \\ más tarde la estrella se me ha detenido \\ muy cerca de San Sebastián.
}

A medida que pasan los años la admiración hacia el arte de este pastor va en aumento. Baste como muestra el gentío que se congregó el 4 de noviembre del 2001 en Urepel, con ocasión de los 25 años de su muerte. Su figura será siempre recordada con cariño y admiración entre los amigos y todos los amantes del bertsolarismo. Como muestra de ello valgan algunos ejemplos.

Su fiel compañero y amigo Mattin le definió como el mejor en versos escritos: "Bertso jartzen, berriz ez zuen parekorik".26 (En cambio no había otro igual a él escribiendo versos). El fino poeta de Laburdi, Iratzeder, resaltó la profundidad de sus versos: "Hain pertxulari sarkorrik ez zen nehun ez nehor". 27 (En ninguna parte hubo un bertsolari tan profundo como él). En las mismas ideas abunda el bertsolari vizcaíno Jon Lopategi quien, además dedicó su "txapela" de campeón el 17 de diciembre de 1989 al pastor de Urepel, en el velódromo de Anoeta en Donostia.

\footnotetext{
Gauza sakonak asmatu eta

gauzak sakon adierazten

Urepeleko maixuagana

ez gera inor irixten.28
}

(No hemos llegado al nivel del maestro de Urepel en la creación de ideas profundas y de la forma íntima de expresarlas.)

Otro de los bertsolaris devotos del arte de Xalbador es el campeón X. Amuriza quien 
en su primer libro de poesía Menditik Mundura (Del monte al mundo, 1977) dedica a Xalbador ocho estrofas de las que escojo una.

\author{
Beste munduko sinesmen finez \\ hots egin zenigun sarri \\ aitortzen dizut hori ez dela \\ niretzat hain kezkagarri. \\ Baldin badago ezin guk kendu \\ ez badago ezin jarri \\ baina egia balitz nahi nuke \\ zure aurrean itzarri \\ betikotasun osoa eman \\ dezagun beha elkarri.29
}

\author{
A menudo nos mostraste \\ tu recia fe en la otra vida \\ te confieso que eso \\ no me resulta tan preocupante. \\ Pues si algo existe no lo podemos negar \\ y si no existe, tampoco podemos crearlo. \\ Pero si fuera verdad, \\ querría despertarme delante de ti \\ para que nos estemos mirando mutuamente \\ durante toda la eternidad.
}

El mundo de la música quiso también asociarse ya a través de coros, ya por medio de voces individuales.30 Aquel sencillo pastor autodidacta que abandonó la escuela francesa porque prefería ser un vasco inculto que un francés ilustrado pero sin euskara, fue propuesto, a título póstumo, como académico correspondiente de Euskaltzaindia - Real Academia de la Lengua Vasca. Otro pastor como él, Erramun Martikorena le describe con cálida voz y con las palabras del poeta y cantautor Xabier Lete.

Adixkide bat bazen orotan bihotz bera

poesiaren hegoek

sentimentuzko bertsoek

antzaldatzen zutena.

Plazetako kantari bakardadez josia

hitzen lihoa iruten

bere barnean irauten

oinazez ikasia.

Nun hago, zer larretan

Urepeleko artzaina, mendi hegaletan gora oroitzapen den gerora ihesetan joan hintzana. (bis)

Hesia urraturik libratu huen kanta,

lotura guztietatik

gorputzaren mugetatik

aski sentitu nahirik.

Azken hatsa huela bertsorik sakonena, inoiz esan ezin diren

estalitako egien

ohiurik bortitzena.31
Érase una vez un amigo de buen corazón

a quien las alas de la poesía

y los bertsos sentidos

le transfiguraban.

Cantor por las plazas abrumado en su soledad

aprendió sufriendo

a hilar el lino de las palabras

a refugiarse en su interior.

¿Dónde estás? ¿En qué prados?

Pastor de Urepel

que ascendiendo las laderas de los montes

has huido hacia el futuro

que es recuerdo.

Rompiendo el cerco liberaste el canto,

queriendo sentirte libre

de todas las ataduras,

de las limitaciones del cuerpo.

Fue tu último suspiro el bertso

el grito más intenso

de las verdades ocultas

que nunca se han podido decir. 
Xalbador murió a los 56 años el 7 de noviembre de 1976. En un homenaje multitudinario celebrado en su pueblo natal, con la doble alegría de la presentación de su libro Odolaren Mintzoa y a los sonidos del Gernikako Arbola dejó de existir este bertsolari lírico y elegíaco a quien el dolor de su pueblo, Euskal Herria, se le convirtió en una dolorida balada.

\section{Década de los 80}

Con el fin de la dictadura franquista acaecida a raíz de la muerte del general Franco, el 20 de noviembre de 1975, llegó a España el período tan ansiado de las nuevas reformas: la Constitución, el parlamento, los partidos políticos, el Estatuto de Autonomía en 1979, la Ley básica de la normalización del uso del euskara en 1982, etc. A duras penas y con bastante lentitud se llegó, por fin, a una situación en la que se podían exhibir sin miedo a castigos, los símbolos políticos como la "ikurriña" (bandera vasca); mostrar públicamente ideas contrarias al gobierno; manifestarse en la calle, etc. Estos vientos de permisividad y libertad obligaron a los bertsolaris a un replanteamiento de la conveniencia del empleo frecuente de los temas políticos en las sesiones bertsolarísticas. Por otra parte, un nuevo público formado especialmente por jóvenes de ambos sexos irrumpió con fuerza en este mundo artístico La televisión vasca (E.T.B) favoreció también eficazmente el desarrollo de esta nueva sensibilidad.

\subsection{Xabier Amuriza. Txapelketas de 1980 y 1982.}

Con el nacimiento de una nueva década, una figura singular surgió en el bertsolarismo: el vizcaíno X. Amuriza (1941 - ). A él se deben, al menos, una serie de cambios como el cuestionamiento de axiomas tradicionales relativos al bertsolarismo; la introducción del vascuence unificado; el uso de nuevas melodías, etc. que supusieron un salto cualitativo muy positivo.

Entre estas novedades, X. Amuriza puso en tela de juicio el axioma tradicionalmente defendido por J. Moulier, "Oxobi” (1888-1958), E. Salaberry (1903 - 1977), Basarri, etc., de que la habilidad demostrada por los vates vascos es un don recibido de Dios, que no se puede adquirir mediante el trabajo y el aprendizaje escolar. Se trata de un don gratuito como el canto de los pájaros.

\footnotetext{
Jainkoak eta bakharrik Jainkoak. Ez da ikasten ibili choria eta khantatzen badaki kantari eskolatu hoberenak baino hobeki. Jitea holakoa du chori horrek eta jitea dena Jainkoak emanik du.32
}

Bertsularien dohaina da [...] Jainkoak emana bertze guztiak bezala Jainkoaren eskutik jalgia.33

Bertsolariak jaio egin behar dik; bere seillu berezia bear dik. Seillu ori ezpadu, berezko ori ezpadu, alper-alperrik alegintzea duk.34

$\mathrm{X}$. Amuriza intentó probar lo contrario afirmando que (supuestas unas condiciones lingüísticas normales de toda persona) el arte bertsolarístico era un problema de esfuerzo y aprendizaje como cualquier otro arte; esta afirmación queda confirmada por los alumnos de 
más de 80 escuelas de bertsolarismo extendidas hoy por Euskal Herria.35 X. Amuriza fue un pionero en la preparación del material pedagógico escolar que realizó durante su larga estancia de más de siete años en la cárcel de Zamora. En dicho material destacan tres libros: Hiztegi errimatua, Hitzaren kirol nazionala (1981) y Zu ere bertsolari (1982) (Tú también bertsolari); este título muestra el objetivo que su autor pretendía probar: que cualquier vasco, con un dominio normal de su lengua, puede llegar a ser bertsolari.

Otra novedad de X. Amuriza en las dos "txapelketak" mencionadas fue el uso del vascuence unificado. Este bertsolari, nacido en Etxano-Zornotza (Bizkaia), es defensor de su dialecto que lo usa siempre que las circunstancias lo requieren. En este aspecto son dignos de mención sus libros: Bizkaiko Bertsogintza (1995, 1998; dos volúmenes); versos tradicionales vizcaínos que han dado origen al disco Vizcayatik....Bizkaiara (2001) del grupo "Oskorri"; Bizkaieraz bertsotan (1996). Los libros de viejas coplas: Bizkaiko Kopla Zaharrak, interpretados musicalmente por Joseba Tapia, así como el disco Katuen Testamentua, cantado también por el mencionado grupo vizcaíno, son algunas de las muestras que manifiestan la querencia de X. Amuriza a su dialecto natal. Pero este bertsolari que es defensor del "euskara batua" desde sus comienzos (1968), al tener que dirigirse a la inmensa mayoría de los vascos se vale del vascuence unificado, como lo demostró en las competiciones nacionales de 1980 y 1982. Como él mismo confiesa, intentó demostrar que este tipo de vascuence avalado por Euskaltzaindia no era "árido y artificial".

La variedad de melodías (algunas fueron creaciones propias) es otro de los aspectos importantes del cambio operado en la década de los 80 . Además de ganar la boina de campeón en estas dos competiciones, X. Amuriza obtuvo también otro primer premio destinado al bertsolari que usara el mayor número de nuevas melodías.

El dramatismo de sus versos, la belleza de su euskara manifestado en diferentes registros, la habilidad de la improvisación demostrada especialmente en las pruebas individuales, y en el tratamiento de temas serios (v.g.: "no solo de pan vive el hombre" o "el padre" de la competición de 1980, así como el tema del "náufrago" en la de 1982 ); la rica gama de recursos retórico-poéticos, etc. son, entre otras aportaciones, las que han jalonado una nueva frontera y han supuesto un notable salto cualitativo en la historia de este fenómeno literario.

El enriquecimiento de rimas, ritmos, nuevas melodías, diferentes enfoques en el tratamiento de los temas, etc. supone también una reflexión profunda en la teorización del bertsolarismo, siguiendo el surco trazado por otros trovadores como Basarri y Xalbador. 36 Sería imposible entender la renovación del bertsolarismo actual sin tener en cuenta la contribución de X. Amuriza en las "txapelketak" de 1980 y 1982. Es una opinión comúnmente aceptada y compartida, entre otros, por el actual campeón A. Egaña quien se siente deudor del artista vizcaíno.

Xabierrek bi gauza garbi irakatsi zizkigun: nora hel gintezkeen eta hara iristeko ze urrats eman behar ziren. Eta horretan, ez ni neu bakarrik, bertsolari eta bertsozale belaunaldi oso bat gatzaizkio zordun. 37 


\section{Década de los 90}

\subsection{Andoni Egaña (1961 - )}

A medida que pasan los años, el bertsolarismo ha ido evolucionando como arte y fenómeno social aunque mantiene aún sus elementos esenciales. Se podría definir como la poesía vasca de estilo oral, por lo general repentizada, que los poetas populares cantan ante el público.

Uno de los representantes más influyentes en esa mutación del bertsolarismo actual es, sin duda, A. Egaña, nacido el 2 de octubre de 1961, en Zarautz (Gipuzkoa). Este bardo vasco se nos muestra como una figura polifacética: bertsolari, escritor galardonado con varios premios, y conferenciante. Supera con ello los parámetros del bertsolari tradicional convirtiéndose en un trovador vasco atípico. Es además un artista que domina con maestría los difíciles recursos del bertsolarismo.

Como escritor ha cultivado la novela, el cuento y el ensayo con obras como: Socratikoek ere badute ama (libro de cuentos, escrito en 1989); Aitaren batean (1990), colección de artículos publicados en la sección "Zabalik" de El Diario Vasco entre los años 1988 y 1990; Zaudete geldi pixka batean (1999); Zozoak beleari (1997), ensayo epistolar en colaboración con J. Sarausa; Imanol Urbieta: Luzea da bidea (2002) y la novela Pausoa noiz luzatu (1998). Ha colaborado en periódicos (el mencionado El Diario Vasco, Euskaldunon egunkaria, Gara, etc.) y en revistas (HABE, Bertsolari, etc.), siendo además guionista y presentador en programas de ETB (Televisión Vasca); es asimismo autor del disco "Tximeletak sabelean" (1994) en el que canta versos vascos.

Como conferenciante y charlista ha participado en cursos organizados por la UPV/ EHU (Universidad del País Vasco / Euskal Herriko Unibertsitatea, universidad pública vasca) en el Palacio Miramar de Donostia, así como en las conferencias dirigidas por la Universidad de Deusto en el salón de la "Kutxa"-Caja de Ahorros de la misma capital. Permítasenos valernos de los recuerdos de la charla que pronunció en ese salón el 7 de setiembre de 1995 en un ciclo de conferencias titulado "Gu geu bertsolari euskaldunok". Con gran interés y sorpresa por parte de los asistentes, A. Egaña presentó varios aspectos de su vida valiéndose de diez fotografías: la infancia en la ikastola "Salbatore Mitxelena" de Zarautz bajo la tutela de profesores como el escritor Andu Lertxundi y el músico I. Urbieta; los tristes recuerdos del fusilamiento de Juan Paredes Manot, "Txiki", y Angel Otaegi, ocurrido en setiembre de 1975, bajo el régimen franquista; la grata impresión que le produjo la victoria de X. Amuriza en la "Txapelketa" de 1980; su carrera universitaria de Filología Vasca comenzada en los EUTG de Donostia y acabada en la UPV/EHU (campus de Vitoria), en la que resalta sus primeros conocimientos teóricos sobre bertsolarismo obtenidos bajo la dirección del afamado oralista de Oiartzun:

Neurri batean bertsotan hastea Juan Mari Lekuonari zor diot. EUTGn Juan Marik ahozko literatura ematen zigun, berarekin oinarri teorikoak indartu nituen.38

Su larga estancia de diez años en tierras alavesas como funcionario cultural del Ayuntamiento de Vitoria, y el regreso a Zarautz como esposo y padre de familia, completaron una parte de esa panorámica biográfica. 
En el transcurso de la conferencia, los oyentes iban conociendo mejor la personalidad de un A. Egaña, tímido por naturaleza, hiperactivo con unas 200 actuaciones bertsolarísticas cada año, consumado deportista a pesar de ser un fumador empedernido:

"Badakizute neure bizio / purua dedala".39

Como bertsolari destacan en él dos facetas: la del diletante y joven Andoni que busca su propia identidad, y la del Egaña maduro, respetado por el prestigio bien logrado como campeón de tres competiciones nacionales: 1993, 1997 y 2001, además de ser finalista en las de 1986 y 1989. Si en la primera faceta aflora el carácter de un joven tímido, en la segunda se observa la fuerte personalidad de un luchador en las lides bertsolarísticas: dotado de reflejos rápidos, poseedor de una inteligencia aguda y de una fina ironía que se convierte en una excelente arma para la controversia dialéctica.

A. Egaña es un bertsolari autodidacta que ha reflexionado pausadamente sobre este fenómeno. Pero es además un trovador ecléctico que ha sabido combinar la influencia del bertsolarismo tradicional del valle del Urola en Gipuzkoa (Joxe Lizaso, J. Agirre "Oranda", I. Lazkano, etc.) con las nuevas aportaciones de un grupo bien avenido de contrastados bertsolaris como Sebastián Lizaso (campeón de Euskadi en 1989), J. Sarasua, A. Peñagarikano (campeón de Gipuzkoa en 1991), I. Murua, X. Euzkitze, M. Mendizabal, M. Tellería, etc. Gracias a esa síntesis, A. Egaña ha logrado que su arte bertsolarístico sea comprendido con facilidad, tanto por los adultos como por los jóvenes aficionados. Su admiración por el fino y humorista bertsolari guipuzcoano, José Miguel Iztueta, "Lazkao-Txiki" (1926-1993) es también patente como lo atestigua la boina de campeón que le brindó en el campeonato de 1993. A. Egaña ha sabido beber en diversas fuentes, logrando así el dominio de diferentes registros y de un estilo muy personal.

Como tantos otros bertsolaris, el bardo de Zarautz es un hombre comprometido con el devenir cultural del País Vasco. Prueba de ello son los bertsos que cantó en Bilbao en honor del poeta G. Aresti, haciéndose eco de la poesía del escritor bilbaíno.

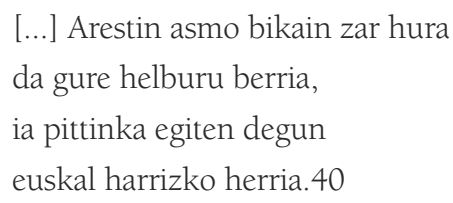

Pero A. Egaña es, ante todo, un bertsolari renovador. Si Basarri sacó el bertsolarimo de las sidrerías para llevarlo a los frontones, y X. Amuriza lo trasladó a las escuelas e ikastolak (escuelas vascas), el bertsolari de Zarautz trata de llevarlo a la universidad, donde aprendió la base teórica de las técnicas de este fenómeno artístico. En sus planes de cambio, A. Egaña marca la necesidad de una profunda renovación en las rimas; este deseo de enriquecer "el almacén de rimas" queda expresado en un bonito símil marinero: "Aingura botata gauden sentzazioa hartuta nago."4l (tengo la impresión de que nos hallamos - como un barco - con el ancla echada).

De las cinco competiciones nacionales en las que tomó parte (1986, 1989, 1993, 1997 y 2001) sugerimos al lector la lectura de varias estrofas: "Artxandatik Bilbora begira" (1986: 148). El saludo inicial dedicado a la mujer, en general, y a la bertsolari Maddalen Lujanbio, en particular (1997: 222). La estrofa dedicada a la cabeza (burua, p. 238); el carterista que intenta 
robar en la "Expo 92" de Sevilla (p. 305); el profesor de 45 años que se enamora de una alumna de 18 ("Txapelketa" 2001: 276-277); el diálogo entre dos madres que han perdido a sus hijos (un ertzaina asesinado por ETA y un militante de esta organización muerto al intentar huir; pp. 301-303).

Hemos afirmado que A. Egaña se siente parte de un grupo de bertsolaris que tratan de adecuar el bertsolarismo a los tiempos modernos. Uno de los frutos maduros de la reflexión llevada a cabo en equipo es el libro El arte del bertsolarismo del que entresaco algunas ideas. 42 La experimentación intelectual es uno de los aspectos más importantes del bertsolarismo actual. El bertsolarismo improvisado del último cuarto de siglo está lleno de textos que confirman la tendencia de los bertsolaris más jóvenes a la experimentación intelectual: X. Amuriza, A. Egaña, J. Sarasua, etc. Para ellos la función poética no es el punto más importante del bertsolarismo improvisado, aunque siga manteniendo su importancia. La expresión corporal, los gestos, son también recursos de primer orden. El bertsolarismo actual se caracteriza por utilizar un gran número de melodías, a diferencia de lo que ocurría en épocas anteriores. También por la cantidad y calidad de actuaciones, autenticidad de la improvisación, nivel organizativo, presencia de creadores jóvenes y la implantación escolar. En cuanto al nivel organizativo, existe el grupo "Euskal Herriko Bertsozale Elkartea" (Asociación de Aficionados al Bertsolarismo del País Vasco) con casi un millar y medio de socios. Sus fines se basan en la transmisión generacional, la documentación-investigación y la organización de la expansión. El bertsolarismo actual cuenta con las bertso-eskolak extendidas por todo el territorio vasco y, desde 1997, con el "Centro de Documentación Xenpelar". La asociación "Bertsozale Elkartea" mantiene relaciones internacionales mediante la participación en encuentros, por ahora, en el ámbito peninsular e iberoamericano. En conclusión, los responsables de la organización del bertsolarismo actual buscan un nuevo marco teórico para el bertsolarismo improvisado. En ese marco la retórica jugará un papel muy importante:

\footnotetext{
"[...] analizar el bertsolarismo como género retórico no supone desterrar por principio

el carácter literario del mismo, por mucho que este carácter literario no constituya un fin en sí".43
}

Como conclusión al bertsolarismo vasco y al bertsolari A. Egaña, presentamos parte de la estrofa que le dedicó X. Amuriza al proclamarse aquel campeón por segunda vez en 1997:

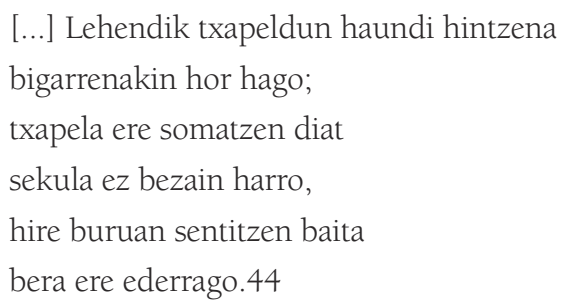

(Tú que ya de antes poseías la boina de campeón, apareces ahí con la segunda; a la boina incluso la noto más orgullosa que nunca pues en tu cabeza se siente ella también más hermosa).

Si X. Amuriza pudo cantar esto con ocasión de la segunda txapela ganada por A.Egaña en 1997, ¿qué no habría podido cantar tras haber logrado la tercera boina en el año 2001, llegando así al nivel del mítico Uztapide? 